\title{
Uterine Malignant Mixed Müllerian Tumors Following Treatment with Selective Estrogen Receptor Modulators in Patients with Breast Cancer: A Report of 13 Cases and Their Clinicopathologic Characteristics
}

\author{
Byung-Kwan Jeong \\ Chang Ohk Sung \\ Kyu-Rae Kim
}

Department of Pathology, Asan Medical Center, University of Ulsan College of Medicine, Seoul, Korea

\begin{abstract}
Background: Breast cancer treatment with selective estrogen receptor modulators (SERMs) increases the incidence of uterine malignant mixed Müllerian tumors (UMMMTS). We examine clinicopathologic characteristics and prognosis of SERM-associated UMMMTs (S-uMMMTs) and discuss possible pathogenetic mechanisms. Methods: Among 28,104 patients with breast cancer, clinicopathologic features and incidence of UMMMT were compared between patients who underwent SERM treatment and those who did not. Of 92 UMMMT cases that occurred during the same period, incidence, dose, and duration of SERM treatment, as well as overall survival rate, were compared for patients with breast cancer who underwent SERM treatment and those who did not (S-UMMMT vs NS-uMMMT) and for patients without breast cancer (de novo-UMMMT). Histopathological findings and immunophenotypes for myogenin, desmin, p53, WT-1, estrogen receptor (ER) $\alpha$, ER $\beta$, progesterone receptor, and GATA-3 were compared between S-UMMMT and de novo-uMMMT. Results: The incidence of S-uMMMT was significantly higher than that of NS-UMMMT (6.35-fold). All patients with SERM were postmenopausal and received daily 20-40 mg SERM. Cumulative SERM dose ranged from 21.9 to $73.0 \mathrm{~g}$ (mean, 46.0) over 39-192 months (mean, 107). Clinicopathologic features, such as International Federation of Gynecology and Obstetrics stage and overall survival, were not significantly different between patients with S-UMMMT and NS-UMMMT or between patients with S-uMMMT and de novo-uMMMT. All 11 S-uMMMT cases available for immunostaining exhibited strong overexpression/null expression of p53 protein and significantly increased ER $\beta$ expression in carcinomatous and sarcomatous components. Conclusions: SERM therapy seemingly increases risk of S-UMMMT development; however, clinicopathologic features were similar in all UMMMTs from different backgrounds. p53 mutation and increased ER $\beta$ expression might be involved in the etiology of S-UMMMT.
\end{abstract}

Key Words: Malignant Müllerian mixed tumor; Selective estrogen receptor modulators; Tamoxifen; Breast neoplasm; p53
The use of selective estrogen receptor modulators (SERMs), including tamoxifen and toremifen, in patients with estrogen receptor (ER)-positive breast cancer is known to cause uterine and ovarian tumors. The therapeutic effect of SERMs in breast cancer is caused by blockade of estrogen binding to ER $\alpha,{ }^{1,2}$ which induces an antiestrogen effect. However, this also produces pro-estrogenic effects in other organ tissues, including the ovaries and endometrium, ${ }^{3-5}$ especially in postmenopausal women. ${ }^{6-11} \mathrm{~A}$ variety of uterine tumors, including endometrial polyps, endometrial hyperplasia, endometrioid adenocarcinoma, Müllerian adenosarcoma, ${ }^{10,12-14}$ endometrial stromal sarcoma, ${ }^{15,16}$ leiomyosarcoma, and uterine malignant mixed Müllerian tumors (uMMMTs), ${ }^{17}$ have been associated with SERM treatment. Some studies have suggested that the relative risk of uMMMTs after tamoxifen treatment is higher than that of endometrial adenocarcinomas, which are well-known secondary malignant tumors associated with tamoxifen treatment. ${ }^{10-13,18}$ Ovarian tumors, including serous cystadenoma, serous cystadenofibroma, fibroma, and endometrioid adenocarcinoma, ${ }^{19}$ as well as small cell carcinoma of pulmonary type ${ }^{20}$ are also known to be associated with SERM treatment, although a causative relationship has not been clearly elucidated. Tamoxifen treatment also induces vascular torsion, cystic necrosis, and ovarian cysts more frequently in premenopausal than postmenopausal women due to supraphysiological level of serum estrogen. ${ }^{21-23}$ For this reason, the current recommendation of the American College of Obstetricians and 
Gynecologists (ACOG) is close monitoring of patients receiving tamoxifen, especially those who are postmenopausal. ${ }^{24}$

The etiology of uterine or ovarian tumors after SERM treatment remains unknown; however, it could be associated with certain factors common to postmenopausal women since most patients are postmenopausal. SERMs or their metabolites may induce a germline or somatic mutation in certain genes, or even epigenetic modifications, ${ }^{25}$ resulting in increased oncogenicity and/or invasiveness.

However, uMMMTs have also been described in patients with BRCA1-associated breast cancer who have not received hormone therapy. ${ }^{26}$ BRCA1 mutation carriers are predisposed to ovarian or uterine papillary serous carcinoma, from which uMMMTs often arise through sarcomatous transformation of highgrade serous carcinomas. ${ }^{27,28}$

As a first step to unveiling the pathogenetic mechanism of uMMMT development after SERM treatment, we studied the incidence and prognosis of UMMMT in patients with breast cancer after SERM treatment, reviewed their clinicopathologic characteristics, and compared histopathological features between uMMMTs with and without SERM treatment and between those that occurred de novo without a prior history of breast cancer. Moreover, we examined the immunohistochemical expression of $\mathrm{ER} \alpha, \mathrm{ER} \beta$, and $\mathrm{p} 53$ to investigate the roles of ER subtype and p53 in the pathogenetic mechanism.

\section{MATERIALS AND METHODS}

\section{Patient selection}

A group of patients with uMMMT but with no breast cancer history was designated as the de novo-uMMMT group. Another group of breast cancer patients who had undergone SERM treatment and later developed uMMMT were designated the SERM-associated uMMMTs (S-uMMMT) group, and breast cancer patients who did not receive SERM treatment but later developed uMMMT were designated the NS-uMMMT group. To analyze the incidence and relative risk of developing S-uMMMT, the prevalence of uMMMT was compared between patients who received SERM treatment and those who did not among 28,104 patients with breast cancer treated at Asan Medical Center, Seoul, Korea, between 2006 and 2016.

Of these patients, 14,221 were treated with SERM. A total of 13 patients developed S-uMMMT, while only two of 13,883 patients who were not treated with SERM developed NS-uMMMT during the same period.

Independently, a list of 92 patients who were histologically diagnosed with uMMMTs during the same study period was retrieved from the database of the Department of Pathology at Asan Medical Center. Patients were divided into two groups based on the presence (13 patients) or absence of a clinical history of breast cancer with SERM treatment (79 patients, de novouMMMTs, including two patients with breast cancer history without SERM treatment). Clinical features for all patients, including age at diagnosis of breast or uterine tumors, menopausal status, International Federation of Gynecology and Obstetrics (FIGO) stage, family history of any malignancies, history of primary malignant tumors in other organs, and follow-up results, were obtained from their medical records. The histological characteristics of S-uMMMTs and NS-uMMMTs were analyzed, including primary site, size of uterine tumors, types of heterologous tumor components, and immunophenotypes. All patients provided written informed consent, and the institutional review board of Asan Medical Center (Seoul, Korea) approved this study (protocol 2018-1154). All study data were obtained in a completely anonymized form.

\section{Histological examination and immunohistochemical staining}

The histopathological features of all 92 patients with uMMMT were reviewed by two pathologists (B.-K.J. and K.-R.K.) with an emphasis on the presence of any particular tumor components.

Tissue sections for immunohistochemical staining were available for 13 of the 15 patients who developed UMMMT with a clinical history of breast cancer. Two of the 15 patients without available tissue sections belonged to the S-uMMMT group.

Paraffin-embedded tissue sections from 13 patients were stained immunohistochemically with mouse monoclonal antibodies to ER $\alpha$ (dilution 1:100, 6F11, Novocastra, Newcastle upon Tyne, UK), ER $\beta$ (dilution 1:400, 14C8, Thermo Fisher Scientific, San Jose, CA, USA), progesterone receptor (PR; 1:200, Novocastra), GATA3 (dilution 1:200, L50-823, Cell Marque, Rocklin, CA, USA), WT-1 (dilution 1:100, 6F-H2, Dako, Glostrup, Denmark), myogenin (1:200, Neomarkers, Fremont, CA, USA), desmin (1:200, D33, Dako), and p53 (1:1,500, DO-7, Dako) using an Autoimmunostainer Benchmark XT (Ventana Medical Systems, Tucson, AZ, USA) and an Optiview DAB IHC detection kit (Ventana Medical Systems). Positive controls were normal endometrial tissue samples for ER $\alpha$ and PR, normal kidney tissue for GATA 3 and WT-1, normal skeletal muscle for desmin, rhabdomyosarcoma tissue for myogenin, and tubal high-grade serous carcinoma for p53. Primary antibodies were omitted for negative controls. 
The immunoreactivity in all slides was scored by Allred's method by considering both the proportion and intensity of positive cells, which were independently recorded for each sample. The proportion of stained cells for ER $\alpha, \operatorname{ER} \beta$, PR, GATA3, WT-1, myogenin, and desmin on each slide was scored using a scale from 0 to 5 , and staining intensity was scored from 0 to 3 . The sum of these two partial scores produced the final score. Zero on this scale indicated negative staining. All cases with a final score $\geq 3$ were considered positive.

A homogeneous staining pattern with strong intensity in more than $80 \%$ of tumor cell nuclei was considered "overexpression" for $\mathrm{p} 53$, while an inhomogeneous and/or weak staining pattern in $<80 \%$ of tumor cell nuclei was considered "nonoverexpression." Complete negativity in any tumor cell nuclei, which is associated with null mutations, ${ }^{29}$ was also considered abnormal. Immunoreactivity in the sarcomatous component was evaluated using the same methods as for the carcinomatous component.

\section{Statistical analysis}

Statistical analysis was performed by SPSS for Windows ver. 23.0 (IBM Corp., Armonk, NY, USA). The odds ratio of uMMMT associated with SERM treatment was estimated by comparing the incidences of S-uMMMT and NS-uMMMT in patients with breast cancer. The comparison was performed using Pearson's chi-square test. Comparison of categorical variables between SuMMMTs and de novo-uMMMTs was also assessed by Pearson's chi-square test, while comparison of quantitative variables was analyzed by Student's t-test. Overall survival was calculated from the date of initial diagnosis until the date of last follow-up or patient death. Overall and recurrence-free survival rates were assessed by the Kaplan-Meier method, and differences between survival curves of patients with S-uMMMT and de novo-uMMMT were compared by the log-rank test. Differences with $\mathrm{p}<$
.05 were considered significant.

\section{RESULTS}

Relative risk of developing uMMMTs in patients with breast cancer after SERM treatment

There was a significant difference in incidence of uMMMT between the two groups of patients with breast cancer (Table 1); a higher proportion of SERM patients developed S-uMMMT ( $\mathrm{n}=$ $13,0.091 \%$ ) compared with those who did not receive SERM treatment $(\mathrm{n}=2,0.014 \%)$, with a 6.35 -fold increased risk of developing S-uMMMT ( $\mathrm{p}=.005)$. The age of patients with breast cancer at diagnosis ranged from 15 to 94 years (median, 53). Patients who did not receive SERM treatment were older than patients with SERM treatment $(\mathrm{p}<.001)$ (Table 2).

Of the 13 patients with SERM, nine were treated with tamoxifen and three were treated with toremifen for 5 years. One was treated with toremifen for the first 4 years and then switched to tamoxifen treatment for uMMMT. The duration of SERM treatment ranged from 36 to 60 months (mean 58 months) with a daily dose of $20-40 \mathrm{mg}$. The cumulative dose of tamoxifen or toremifen ranged from 21.9 to $73.0 \mathrm{~g}$ (mean, $46.0 \mathrm{~g}$; median, $36.5 \mathrm{~g})$.

Of the 10 patients with S-uMMMT whose breast cancer tissue was available for p53 immunostaining, eight exhibited a wildtype pattern, while two exhibited strong reactivity. In contrast, strong immunoreactivity or complete negativity was present in all 11 patients whose S-uMMMT tissue was available for p53 immunostaining, suggesting that a new p 53 mutation had occurred in the uterine tumor.

\section{Clinicopathologic features of S-uMMMTs}

The age of patients with breast cancer who later developed SuMMMT ranged from 37 to 67 years (mean, 53), and all but one

Table 1. Incidence of UMMMTs in patients with breast cancer who did or did not undergo preceding long-term SERM treatment

\begin{tabular}{lccc}
\hline & UMMMT, $n(\%)$ & Odds ratio & $p$-value \\
\hline Breast cancer treated with long-term SERM & $13 / 14,221(0.091)$ & 6.350 & .004 \\
Breast cancer treated without long-term SERM & $2 / 13,883(0.014)$ & & \\
\hline
\end{tabular}

UMMMTs, uterine malignant mixed Müllerian tumors; SERM, selective estrogen receptor modulator.

Table 2. Comparison of age in patients with breast cancer who did or did not undergo preceding long-term SERM treatment

\begin{tabular}{lcc}
\hline & Median age and older ( $\geq 53$ yr) & Younger than median age $(<53$ yr) \\
\hline Breast cancer treated with long-term SERM & $6,911(48.6)$ & $7,310(51.4)$ \\
Breast cancer treated without long-term SERM & $7,650(55.1)$ & $6,233(44.9)$ \\
\hline
\end{tabular}

Values are presented as number (\%).

SERM, selective estrogen receptor modulator. 
patient $(\mathrm{n}=12,92.3 \%)$ were postmenopausal (Table 3). The age at diagnosis of S-uMMMT ranged from 40 to 76 years (mean, 62), at which point all were menopausal or postmenopausal. The duration from start of SERM treatment to onset of S-uMMMT ranged from 39 to 192 months (mean, 107 months).

The size of S-uMMMTs ranged from 1.5 to $13 \mathrm{~cm}$ in longest dimension (mean, $6.8 \mathrm{~cm}$ ). Three patients $(23.1 \%)$ had lymph node metastasis at the time of diagnosis of S-uMMMT, and eight patients (53.8\%) had lymphovascular invasion. Seven patients had FIGO stage I (53.8\%), three had stage III (23.1\%), and three had stage IV (23.1\%).
BRCA mutation tests were not performed in any patients; however, none of the 13 patients with S-uMMMT had any family history of breast, ovarian, or endometrial cancers. One of the two NS-uMMMT patients had a family history of breast and endometrial cancers in her siblings.

Clinical outcomes including lymph node metastasis $(\mathrm{p}=.694)$, FIGO stage $(\mathrm{p}=.518)$, overall survival rate $(\mathrm{p}=.859)$, and recurrence-free survival rate $(\mathrm{p}=.696)$ were not significantly different between patients with S-uMMMTs and de novo-uMMMTs (Table 4, Fig. 1), nor between patients with S-uMMMT and NS-uMMMT. The rate of lymph node metastasis was slightly lower in

Table 3. Clinicopathologic characteristics of patients with uterine malignant mixed Müllerian tumor with preceding breast cancer history

\begin{tabular}{|c|c|c|c|c|c|c|c|c|}
\hline Age (yr) & $\begin{array}{l}\text { SERM } \\
\text { treatment }\end{array}$ & $\begin{array}{l}\text { Time from treatment } \\
\text { to onset (mo) }\end{array}$ & Menopause & $\begin{array}{l}\text { Tumor size (greatest } \\
\text { dimension in cm) }\end{array}$ & $\begin{array}{l}\text { Lymph node } \\
\text { metastasis }\end{array}$ & $\begin{array}{l}\text { Lymphovascular } \\
\text { invasion }\end{array}$ & $\begin{array}{l}\text { FIGO } \\
\text { stage }\end{array}$ & $\begin{array}{l}\text { Sarcomatous } \\
\text { component }\end{array}$ \\
\hline 72 & TOR $5 \mathrm{yr}$ & 61 & Postmenopause & 6.5 & Not identified & Not identified & $1 \mathrm{~A}$ & Heterologous \\
\hline 68 & TAM 5 yr & 48 & Postmenopause & 1.5 & Not identified & Not identified & $1 \mathrm{~B}$ & Homologous \\
\hline 52 & TAM 5 yr & 61 & Postmenopause & 2.8 & Present & Present & $3 \mathrm{C} 2$ & Homologous \\
\hline 40 & TAM 3 yr & 39 & Postmenopause & 10.9 & Not identified & Present & $3 \mathrm{~A}$ & Homologous \\
\hline 58 & TAM $5 \mathrm{yr}$ & 119 & Postmenopause & 6.0 & Not identified & Present & $3 \mathrm{~A}$ & Homologous \\
\hline 59 & TAM 5 yr & 128 & Postmenopause & 25.0 & Present & Present & $4 \mathrm{~B}$ & Heterologous \\
\hline 69 & TOR $5 \mathrm{yr}$ & 107 & Postmenopause & 6.8 & Not identified & Not identified & $1 \mathrm{~A}$ & Homologous \\
\hline 53 & TAM 5 yr & 69 & Premenopause & 13.0 & Present & Present & $4 \mathrm{~B}$ & Heterologous \\
\hline 61 & TAM 5 yr & 115 & Postmenopause & 7.5 & Not identified & Not identified & $4 \mathrm{~B}$ & Heterologous \\
\hline 55 & TAM 5 yr & 77 & Postmenopause & 1.5 & Not identified & Not identified & $1 \mathrm{~A}$ & Homologous \\
\hline 75 & TAM 5 yr & 178 & Postmenopause & 8.0 & Not identified & Present & $1 \mathrm{~A}$ & Heterologous \\
\hline 54 & No & 60 & Postmenopause & 2.4 & Not resected ${ }^{\mathrm{a}}$ & Not identified & $1 \mathrm{~A}$ & Heterologous \\
\hline 65 & No & 65 & Postmenopause & 9.0 & Not identified & Present & 4B & Heterologous \\
\hline
\end{tabular}

SERM, selective estrogen receptor modulator; FIGO, International Federation of Gynecology and Obstetrics; TOR, toremifen; TAM, tamoxifen.

aLymph nodes were preserved.

Table 4. Comparison of clinicopathologic characteristics between patients with uMMMT with preceding SERM treatment (S-uMMMT) and patients with de novo-uMMMT

\begin{tabular}{|c|c|c|c|c|}
\hline Baseline characteristic & Total $(n=92)$ & S-uMMMT $(n=13)$ & De novo-uMMMT $(n=79)$ & $p$-value \\
\hline & & & & .555 \\
\hline Patient age (yr) & $61(40-77)$ & $60(40-76)$ & $60(45-77)$ & \\
\hline Menopause & & & & .523 \\
\hline Premenopause & $4(4.3)$ & $1(7.7)$ & $3(3.8)$ & \\
\hline Postmenopause & $88(95.7)$ & $12(92.3)$ & 76 (96.2) & \\
\hline Tumor size (greatest dimension) & & & & .404 \\
\hline Size $(\mathrm{cm})$ & $6(0.6-15.2)$ & $6.8(1.5-13.0)$ & $6(0.6-15.2)$ & \\
\hline Node metastasis & $37(40.2)$ & $3(23.1)$ & $34(45.9)$ & .174 \\
\hline Lymphovascular invasion & $52(56.5)$ & $8(61.5)$ & $44(55.7)$ & .694 \\
\hline FIGO stage & & & & .518 \\
\hline Stage 1 & $37(40.7)$ & $7(53.8)$ & $30(38.5)$ & \\
\hline Stage 2 & $5(5.5)$ & 0 & $5(6.4)$ & \\
\hline Stage 3 & $32(35.2)$ & $3(23.1)$ & $29(37.1)$ & \\
\hline Stage 4 & $17(18.7)$ & $3(23.1)$ & $14(17.9)$ & \\
\hline Heterologous sarcomatous component & $34(37.0)$ & $5(38.5)$ & $29(36.7)$ & .903 \\
\hline
\end{tabular}

Values are presented as median (range) or number (\%)

UMMMT, uterine malignant mixed Müllerian tumor; SERM, selective estrogen receptor modulator; FIGO, International Federation of Gynecology and Obstetrics. aBased on a chi-square test for categorical variables and on a t-test for continuous variables. 

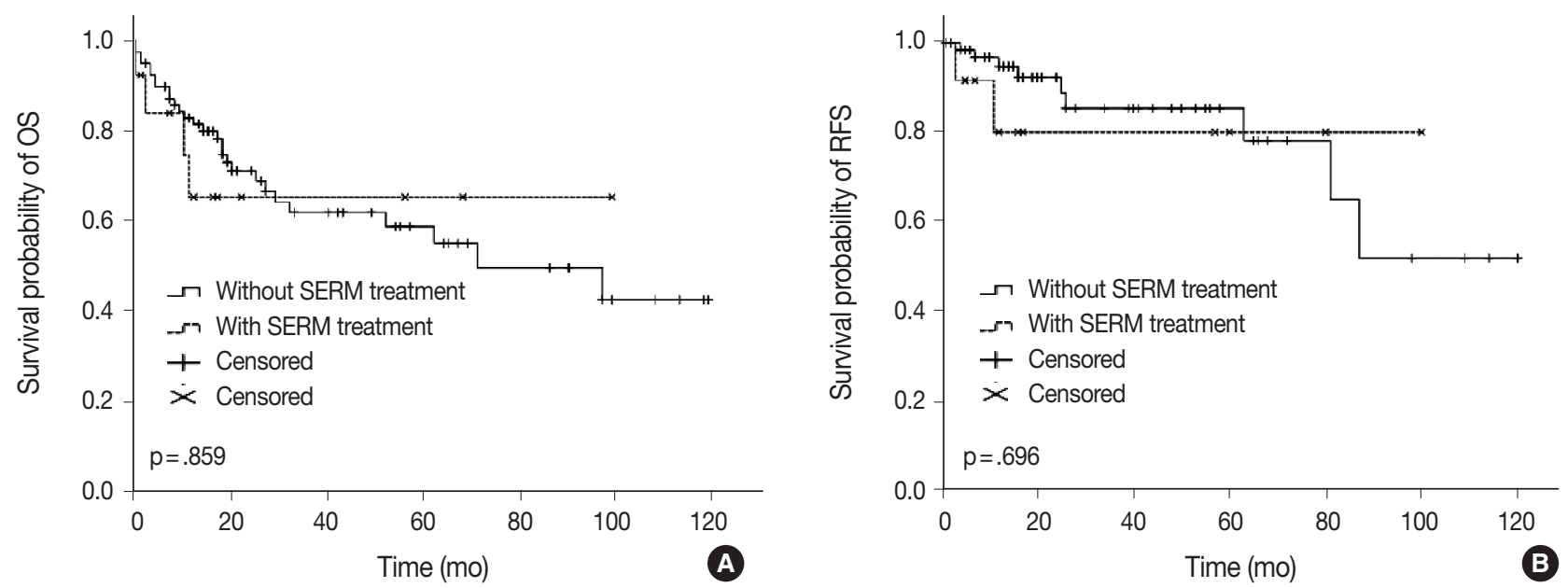

Fig. 1. Kaplan-Meier survival curves comparing overall survival (OS) (A) and recurrence-free survival (RFS) (B) rates in patients with selective estrogen receptor modulators (SERM)-treated breast cancer with subsequent uterine malignant mixed Müllerian tumors (uMMMTs) and de novo-uMMMTs in patients with no preceding breast cancer or preceding breast cancer without SERM treatment.

patients with S-uMMMT compared to those with de novo-uMMMT (23.1\% vs $45.9 \%)$, but the difference was not statistically significant $(\mathrm{p}=.174)$.

\section{Histological comparison of UMMMTs in breast cancer patients with and without SERM treatment}

Histologically, all 13 S-uMMMTs were composed of a mixture of malignant epithelial and mesenchymal components. Rhabdomyoblasts were frequently identified in hematoxylin and eosin-stained sections in five patients (38.5\%), and the exclusive presence of rhabdomyoblasts was a characteristic of one patient, mimicking a pure rhabdomyosarcoma. Other components such as chondrosarcomatous, osteosarcomatous, or liposarcomatous components were not identified. Of the 79 de novo-uMMMT, 50 were homologous, while 29 (36.7\%) contained heterologous elements in the form of rhabdomyoblasts (18 cases, 22.8\%) or malignant cartilage (8 cases, $10.1 \%)$. Epithelial components were mostly composed of high-grade papillary serous carcinomas both in S-uMMMTs and de novo-uMMMTs, and serous carcinoma was the only epithelial component in seven cases (53.8\%). Three cases of S-uMMMT (23.1\%) exhibited sarcomatous overgrowth with a hardly discernible carcinomatous component, while this feature was found in eight cases of de novo-uMMMT (10.1\%). Collectively, significant histopathological differences were not identified between S-uMMMTs and de novo-uMMMTs.

\section{Immunohistochemistry of UMMMT cases with preceding} breast cancer

Protein expression as revealed by immunohistochemical staining did not show any significant differences between S-uMMMTs ( $\mathrm{n}=$ 11) and NS-uMMMTs ( $\mathrm{n}=2$ ) (Fig. 2). All 11 S-uMMMT samples exhibited either diffuse overexpression $(n=8)$ or complete loss of p53 expression $(n=3)$ in the nuclei.

All 11 S-uMMMT cases exhibited strong immunoreactivity for ER $\beta$ in both carcinomatous and sarcomatous components (Fig. 2). Only $45.5 \%$ of cases $(n=5)$ showed weak, focal immunoreactivity to $E R \alpha$, and the remaining cases were negative, suggesting an increased ratio of ER $\beta$ to ER $\alpha$ expression. Progesterone receptors were expressed in a smaller number of cases $(\mathrm{n}=2)$, while the frequency of immunoreactivity to myogenin and desmin, which highlight rhabdomyoblasts, was $45.5 \%(\mathrm{n}=$ $5)$ and $45.5 \%(n=5)$, respectively. Detailed information of immunohistochemical staining results is presented in Table 5 .

\section{DISCUSSION}

The occurrence of uMMMTs has been described in patients with BRCA1-associated breast cancer who did not receive hormone therapy, as well as in patients with SERM treatment. ${ }^{26}$ Moreover, one population-based study proposed that hormone therapy itself has a negligible effect on the incidence of uMMMT. ${ }^{26}$ Therefore, we initially suspected that oncogenicity after SERM treatment could be associated with preexisting genetic changes, such as BRCA mutations. However, none of the $13 \mathrm{~S}$ uMMMT patients in our cohort had a family history of malignant tumors, especially in breasts and female genital organs. In addition, most patients were postmenopausal at the time of breast or uterine cancer diagnosis, which is not a typical clinical 
feature of BRCA mutation-associated tumors. It is less likely that patients with S-uMMMT have a hereditary cancer predisposition, although comprehensive genetic analysis was not per- formed in this study. Patients with breast cancer who were treated with SERM were younger than those who were not treated with SERM. This result is not consistent with previous studies
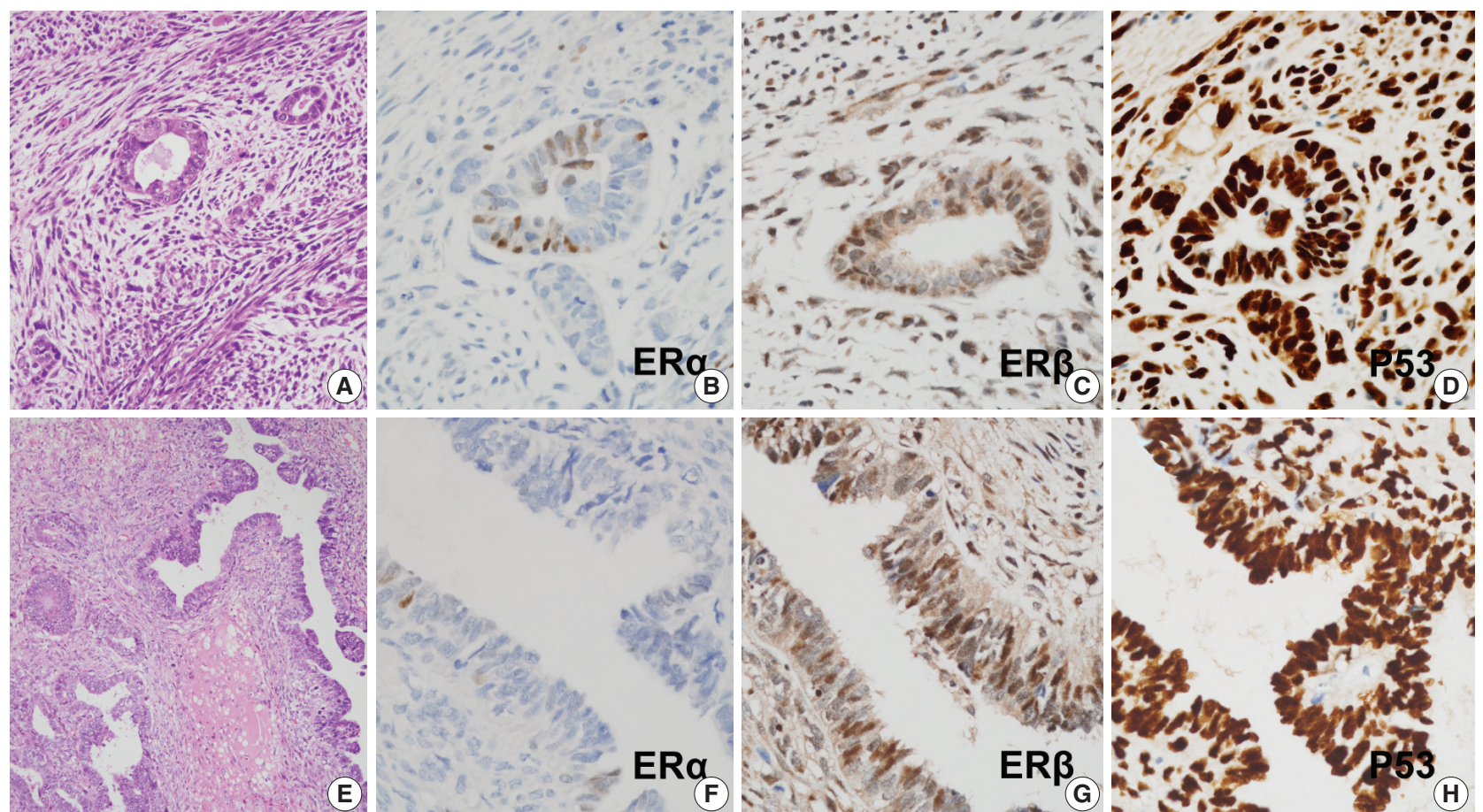

Fig. 2. Histopathologic and immunohistochemical features of uterine malignant mixed Müllerian tumors (A-D) occurring in patients with breast cancer with $(A-D)$ or without $(E-H)$ prior selective estrogen receptor modulator treatment, showing no significant differences: hematoxylin and eosin (A, E), estrogen receptor (ER) $\alpha(B, F)$, ERß (C, G), and p53 (D, H).

Table 5. Allred scoring of immunohistochemical results of patients with uMMMT with preceding breast cancer

\begin{tabular}{|c|c|c|c|c|c|c|c|c|c|}
\hline Patient & Allred score & $\mathrm{ER} \alpha$ & $\mathrm{ER} \beta$ & PR & GATA-3 & WT-1 & p53 & Myogenin & Desmin \\
\hline \multicolumn{10}{|l|}{ SERM (+) } \\
\hline \multirow[t]{2}{*}{ Negative } & 0 & $6(54.5)$ & - & $9(81.8)$ & $11(100)$ & $1(9.1)$ & $3(27.3)^{a}$ & $6(54.5)$ & $6(54.5)$ \\
\hline & 2 & - & - & - & - & - & - & - & - \\
\hline \multirow[t]{6}{*}{ Positive } & 3 & - & - & - & - & - & - & - & - \\
\hline & 4 & $2(18.2)$ & - & - & - & - & - & - & - \\
\hline & 5 & $1(9.1)$ & - & - & - & $3(27.3)$ & - & $4(36.4)$ & $4(36.4)$ \\
\hline & 6 & - & $2(18.2)$ & - & - & $4(36.4)$ & - & $1(9.1)$ & $1(9.1)$ \\
\hline & 7 & $2(18.2)$ & 7 (63.6) & $2(18.2)$ & - & - & - & - & - \\
\hline & 8 & - & $2(18.2)$ & - & - & $3(27.3)$ & $8(72.7)$ & - & - \\
\hline \multicolumn{10}{|l|}{ SERM (-) } \\
\hline \multirow[t]{2}{*}{ Negative } & 0 & - & - & $1(50)$ & $2(100)$ & - & - & - & - \\
\hline & 2 & - & - & - & - & - & - & - & - \\
\hline \multirow[t]{6}{*}{ Positive } & 3 & - & - & - & - & - & - & - & - \\
\hline & 4 & - & - & - & - & - & - & $2(100)$ & $2(100)$ \\
\hline & 5 & $2(100)$ & - & $1(50)$ & - & - & - & - & - \\
\hline & 6 & - & $1(50)$ & - & - & $2(100)$ & - & - & - \\
\hline & 7 & - & $1(50)$ & - & - & - & - & - & - \\
\hline & 8 & - & - & - & - & - & $2(100)$ & - & - \\
\hline
\end{tabular}

Values are presented as number (\%).

UMMMT, uterine malignant mixed Müllerian tumor; ER, estrogen receptor; PR, progesterone receptor; SERM, selective estrogen receptor modulator. ${ }^{a}$ All tumor cell nuclei were negative. 
showing that triple negative breast cancers are diagnosed at a younger age than ER and/or PR positive breast cancers. ${ }^{30,31}$ This might be related to the methodology of the present study, which only reflects SERM treatment status, while exact statuses of ER, PR, and HER2 were not collected. Our results suggest that differences in menopause status due to age did not alter the effect of SERM treatment on uMMMT.

Endometrial carcinomas develop in females who have had cumulative doses greater than $35 \mathrm{~g}$ of tamoxifen; ${ }^{32}$ however, only a small proportion of patients who received this amount of drug developed uterine malignancy in this study, irrespective of dose. The duration of SERM treatment ranged widely from 39 to 192 months (mean, 107 months), so dose and duration may not be closely related to risk factors of uMMMT.

While endometrial carcinomas after tamoxifen treatment are equally distributed in pre- and postmenopausal women, ${ }^{33} \mathrm{~S}$ uMMMT occurs almost exclusively in postmenopausal women in both this study and in the literature. ${ }^{17,34}$ Thus, the tumorigenic mechanism of S-uMMMT could be associated with certain factors common to postmenopausal women. One cross-sectional study reported that ER $\beta$ expression, but not $\mathrm{ER} \alpha$, was higher in postmenopausal women than in premenopausal women, ${ }^{35}$ suggesting that $\operatorname{ER} \beta$ expression or the ratio of $\operatorname{ER} \beta$ to $\operatorname{ER} \alpha$ is elevated in postmenopausal women. $\operatorname{ER} \alpha$ and $\operatorname{ER} \beta$ have similarities in structure and mechanism of action, including interaction with other transcription factors, but they have distinct functions in hormonal resistance and cellular processes.

Resistance to endocrine therapy develops in 25\%-50\% of patients who receive tamoxifen treatment for breast cancer, and the mechanism by which this occurs has been partly elucidated. Tamoxifen disrupts estrogen-ER $\alpha$ binding and can block cell proliferation and induce apoptotic cell death. During tamoxifen treatment, ubiquitin ligase CUE domain-containing protein 2 (CUEDC2) is expressed at low levels, which causes a low rate of proteasome-mediated degradation of ER $\alpha{ }^{36}$ Tamoxifen-resistant cells with increased expression of CUEDC2 may appear, thereby elevating degradation and turnover of $\mathrm{ER} \alpha$ and conferring resistance to tamoxifen treatment. ${ }^{36}$ The inhibitory effect of $\operatorname{ER} \beta$ on binding of p53-ER $\alpha$ suggests that competition between ER subtypes on the binding of p53 may occur in cells that express both receptors. While there are inverse associations between ER $\alpha$ activity and $\mathrm{p} 53, \mathrm{ER} \beta$ has been reported to inhibit breast tumorigenesis by acting alone or in concert with $\mathrm{p} 53$ and $\mathrm{p} 63 .{ }^{37}$ In our study, all 11 cases of S-uMMMT available for immunostaining exhibited diffuse, strong overexpression for $\mathrm{p} 53$ and ER $\beta$ and negative or weak expression for ER $\alpha$ and PR in both carcinomatous and sarcomatous components. Further research is needed to clarify how SERM works on $\operatorname{ER} \beta$, especially in postmenopausal women.

In conclusion, the immunohistochemical results in our study suggest that both S-uMMMTs and NS-uMMMTs are related to p53 mutation, and clinicopathologic features were similar in all uMMMTs from different backgrounds. Increased ER $\beta$ expression or ratio of $\operatorname{ER} \beta$ to $E R \alpha$ in postmenopausal women and binding of ER $\beta$ to mutant $\mathrm{p} 53$ might induce carcinogenesis, sarcomatous transformation, epithelial mesenchymal transition, and invasiveness in organ tissues expressing both receptors. This hypothesis should be tested in further detailed studies.

\section{ORCID}

Byung-Kwan Jeong: https://orcid.org/0000-0002-0038-3959

Chang O. Sung: https://orcid.org/0000-0002-8567-456X

Kyu-Rae Kim: https://orcid.org/0000-0002-5781-7447

\section{Conflicts of Interest}

The authors declare that they have no potential conflicts of interest.

\section{REFERENCES}

1. Kennedy BJ. Hormone therapy for advanced breast cancer. Cancer 1965; 18: $1551-7$.

2. Elwood JM, Godolphin W. Oestrogen receptors in breast tumours: associations with age, menopausal status and epidemiological and clinical features in 735 patients. Br J Cancer 1980; 42: 635-44.

3. Cook LS, Weiss NS, Schwartz SM, et al. Population-based study of tamoxifen therapy and subsequent ovarian, endometrial, and breast cancers. J Natl Cancer Inst 1995; 87: 1359-64.

4. Webb P, Lopez GN, Uht RM, Kushner PJ. Tamoxifen activation of the estrogen receptor/AP-1 pathway: potential origin for the cellspecific estrogen-like effects of antiestrogens. Mol Endocrinol 1995; 9: 443-56.

5. Assikis VJ, Jordan VC. Gynecologic effects of tamoxifen and the association with endometrial carcinoma. Int J Gynaecol Obstet 1995; 49: 241-57.

6. van Leeuwen FE, Benraadt J, Coebergh JW, et al. Risk of endometrial cancer after tamoxifen treatment of breast cancer. Lancet 1994; 343: 448-52.

7. Fisher B, Costantino JP, Redmond CK, Fisher ER, Wickerham DL, Cronin WM. Endometrial cancer in tamoxifen-treated breast cancer patients: findings from the National Surgical Adjuvant Breast and 
Bowel Project (NSABP) B-14. J Natl Cancer Inst 1994; 86: 527-37.

8. Barakat RR. The effect of tamoxifen on the endometrium. Oncology (Williston Park) 1995; 9: 129-34.

9. Bernstein L, Deapen D, Cerhan JR, et al. Tamoxifen therapy for breast cancer and endometrial cancer risk. J Natl Cancer Inst 1999; 91: 1654-62.

10. Bergman L, Beelen ML, Gallee MP, Hollema H, Benraadt J, van Leeuwen FE. Risk and prognosis of endometrial cancer after tamoxifen for breast cancer. Comprehensive Cancer Centres' ALERT Group. Assessment of liver and endometrial cancer risk following tamoxifen. Lancet 2000; 356: 881-7.

11. Jones ME, van Leeuwen FE, Hoogendoorn WE, et al. Endometrial cancer survival after breast cancer in relation to tamoxifen treatment: pooled results from three countries. Breast Cancer Res 2012; 14: R91.

12. Wysowski DK, Honig SF, Beitz J. Uterine sarcoma associated with tamoxifen use. N Engl J Med 2002; 346: 1832-3.

13. Wickerham DL, Fisher B, Wolmark N, et al. Association of tamoxifen and uterine sarcoma. J Clin Oncol 2002; 20: 2758-60.

14. Lavie O, Barnett-Griness O, Narod SA, Rennert G. The risk of developing uterine sarcoma after tamoxifen use. Int J Gynecol Cancer 2008; 18: 352-6.

15. Eddy GL, Mazur MT. Endolymphatic stromal myosis associated with tamoxifen use. Gynecol Oncol 1997; 64: 262-4.

16. Christie DB 3rd, Day JD, Moore AB, Chapman JR, Nakayama DK, Conforti AM. Endometrial stromal sarcoma development after hysterectomy and tamoxifen therapy. Am Surg 2008; 74: 726-8.

17. McCluggage WG, Abdulkader M, Price JH, et al. Uterine carcinosarcomas in patients receiving tamoxifen: a report of 19 cases. Int J Gynecol Cancer 2000; 10: 280-4.

18. Curtis RE, Freedman DM, Sherman ME, Fraumeni JF Jr. Risk of malignant mixed mullerian tumors after tamoxifen therapy for breast cancer. J Natl Cancer Inst 2004; 96: 70-4.

19. Cohen I, Altaras MM, Lew S, Tepper R, Beyth Y, Ben-Baruch G. Ovarian endometrioid carcinoma and endometriosis developing in a postmenopausal breast cancer patient during tamoxifen therapy: a case report and review of the literature. Gynecol Oncol 1994; 55(3 Pt 1): 443-7.

20. Yin L, Li J, Wei Y, Ma D, Sun Y, Sun Y. Primary ovarian small cell carcinoma of pulmonary type with coexisting endometrial carcinoma in a breast cancer patient receiving tamoxifen: a case report and literature review. Medicine (Baltimore) 2018; 97: e10900.

21. Shushan A, Peretz T, Uziely B, Lewin A, Mor-Yosef S. Ovarian cysts in premenopausal and postmenopausal tamoxifen-treated women with breast cancer. Am J Obstet Gynecol 1996; 174(1 Pt 1): $141-4$.
22. Cohen I, Figer A, Tepper R, et al. Ovarian overstimulation and cystic formation in premenopausal tamoxifen exposure: comparison between tamoxifen-treated and nontreated breast cancer patients. Gynecol Oncol 1999; 72: 202-7.

23. Kojima N, Yamasaki Y, Koh H, Miyashita M, Morita H. Long-acting luteinizing hormone-releasing hormone agonist for ovarian hyperstimulation induced by tamoxifen for breast cancer. Case Rep Obstet Gynecol 2018; 2018: 4931852.

24. Committee Opinion No. 601: Tamoxifen and uterine cancer. Obstet Gynecol 2014; 123: 1394-7.

25. Pandey V, Zhang M, Chong QY, et al. Hypomethylation associated enhanced transcription of trefoil factor-3 mediates tamoxifen-stimulated oncogenicity of ER+ endometrial carcinoma cells. Oncotarget 2017; 8: 77268-91.

26. Wilson BT, Cordell HJ. Uterine carcinosarcoma/malignant mixed Mullerian tumor incidence is increased in women with breast cancer, but independent of hormone therapy. J Gynecol Oncol 2015; 26: 249-51.

27. Biron-Shental T, Drucker L, Altaras M, Bernheim J, Fishman A. High incidence of BRCA1-2 germline mutations, previous breast cancer and familial cancer history in Jewish patients with uterine serous papillary carcinoma. Eur J Surg Oncol 2006; 32: 1097-100.

28. Pennington KP, Walsh T, Lee $\mathrm{M}$, et al. BRCA1, TP53, and CHEK2 germline mutations in uterine serous carcinoma. Cancer 2013; 119: 332-8.

29. Yemelyanova A, Vang R, Kshirsagar M, et al. Immunohistochemical staining patterns of $\mathrm{p} 53$ can serve as a surrogate marker for TP53 mutations in ovarian carcinoma: an immunohistochemical and nucleotide sequencing analysis. Mod Pathol 2011; 24: 1248-53.

30. Martinez ME, Wertheim BC, Natarajan L, et al. Reproductive factors, heterogeneity, and breast tumor subtypes in women of mexican descent. Cancer Epidemiol Biomarkers Prev 2013; 22: 1853-61.

31. Song N, Choi JY, Sung H, et al. Heterogeneity of epidemiological factors by breast tumor subtypes in Korean women: a case-case study. Int J Cancer 2014; 135: 669-81.

32. Ismail SM. Pathology of endometrium treated with tamoxifen. J Clin Pathol 1994; 47: 827-33.

33. Swerdlow AJ, Jones ME; For the British Tamoxifen Second Cancer Study Group. Tamoxifen treatment for breast cancer and risk of endometrial cancer: a case-control study. J Natl Cancer Inst 2005; 97: 375-84.

34. Vasconcelos AL, Nunes B, Duarte C, et al. Tamoxifen in breast cancer ipse dixit in uterine malignant mixed Mullerian tumor and sarcoma: a report of 8 cases and review of the literature. Rep Pract Oncol Radiother 2013; 18: 251-60.

35. McInnes KJ, Andersson TC, Simonyte K, et al. Association of 11beta- 
hydroxysteroid dehydrogenase type I expression and activity with estrogen receptor beta in adipose tissue from postmenopausal women. Menopause 2012; 19: 1347-52.

36. Thomas C, Gustafsson JA. A CUE hints at tumor resistance. Nat Med 2011; 17: 658-60.
37. Bado I, Nikolos F, Rajapaksa G, Gustafsson JÅ, Thomas C. ERbeta decreases the invasiveness of triple-negative breast cancer cells by regulating mutant p53 oncogenic function. Oncotarget 2016; 7: 13599-611. 